\title{
Evolución de la calidad de vida en pacientes en hemodiálisis: Estudio prospectivo a un año
}

\author{
Rosa Muñoz Sancho \\ Ana Oto Royo \\ Rosa Barrio Alonso \\ Manuel Fernández
}

\author{
Servicio de Nefrología \\ Hospital Universitario Miguel Servet. \\ Zaragoza
}

\section{RESUMEN}

Antecedentes. En un estudio previo describimos como la calidad de vida $(\mathrm{CV})$ en pacientes en hemodiálisis está deteriorada respecto a la población general sana y que el estado depresivo y el grado de comorbilidad son los factores determinantes más significativos de la $\mathrm{CV}$. En el presente estudio se evalúan nuevamente a los mismos pacientes tras un año de evolución y se estudian los factores determinantes de los cambios en el nivel de CV. Métodos. Se incluyeron todos los pacientes estudiados un año antes y se pasaron test de calidad de vida (SF-36) y test psicológicos. Se registró el grado de comorbilidad, los datos demográficos y los eventos de morbilidad. Resultados. La serie para el análisis quedó constituida por 52 pacientes, tras excluir a los que se habían trasplantado y a los fallecidos. Solo el componente de funcionalismo físico se redujo de forma significativa mientras que el resto de los componentes no varió. El grado de ansiedad y depresión no mostró diferencias en un año de intervalo. El valor de la $\mathrm{CV}$ viene determinado de forma significativa por el estado de depresión, la edad y el grado de comorbilidad. En conjunto, estas 3 variables justifican el 67\% de la variabilidad del dominio función física.

PALABRAS CLAVE: CALIDAD DE VIDA HEMODIÁLIS

\section{Correspondencia \\ Rosa Muñoz Sancho \\ Hemodiálisis Planta Baja \\ Hospital Miguel Servet \\ Isabel la Católica, 1 \\ 50009 Zaragoza \\ e-mail: sant422@separ.es}

\section{EVOLUTION OF THE QUALITY OF LIFE IN PATIENTS ON HAEMODIALYSIS: ONE-YEAR PROSPECTIVE STUDY}

\section{SUMMARY}

Background. In a previous study we described how the quality of life (QL) in patients undergoing haemodialysis is lower compared to the general healthy population and that the depressive state and degree of comorbidity are the most significant QL determining factors. This study carries out a new assessment of the same patients after one year and studies the factors determining changes in QL level. Methods. All the patients studied one year earlier were included and underwent quality of life tests (SF-36) and psychological tests. The degree of comorbidity was recorded, as well as demographical data and morbidity events. Results. The series for analysis was made up of 52 patients, after excluding those who had received transplants and those who had died. Only the physical functionalism component was reduced significantly while the rest of the components did not vary. The degree of anxiety and depression does not show differences in an interval of one year. The value of the QL is determined to a significant extent by the state of depression, age and the degree of comorbidity. Taken together, these 3 variables account for $67 \%$ of the variability in the physical function domain.

\section{KEWORDS QUALITY OF LIFE} HAMEODIALYSIS 


\section{INTRODUCCIÓN}

En un estudio previo describimos como la calidad de vida (CV) en pacientes en hemodiálisis, analizada mediante un instrumento genérico como el SF-36, está claramente reducida respecto a la población general ${ }^{1}$. Encontramos que la pérdida de $\mathrm{CV}$ no es homogénea y no depende del sexo, del estado nutricional ni del tiempo en hemodiálisis. La determinación de la calidad de vida en el tiempo se ha convertido en un factor a medir en respuesta a una intervención médica o de enfermería. En estudios previos realizados en nuestro país, se ha encontrado un claro deterioro de la calidad de vida en pacientes en hemodiálisis y un mayor nivel del estado de ansiedad y depresión ${ }^{2}$. En uno de estos estudios se encontró una relación inversa y significativa entre el estado de ansiedad-depresión y la calidad de vida. Sin embargo en este estudio solo se relacionó estas dos variables en el grupo de pacientes con más afectación en su calidad de vida y el número total de casos era escaso.

\section{OBJETIVOS}

El objetivo principal es evaluar los cambios en el nivel de calidad de vida percibido por pacientes en hemodiálisis a lo largo del tiempo, en este caso a un año, en el contexto de un estudio prospectivo sobre la calidad de vida en este tipo de patología. Los objetivos adicionales son: establecer los determinantes demográficos, del estado de ansiedad/depresión y los cambios de morbilidad en las variaciones anuales del nivel de CV.

\section{MÉTODOS}

Pacientes. En el estudio se incluyeron todos los pacientes estudiados un año antes y que seguían acudiendo a nuestra Unidad de Hemodiálisis. A lo largo del año de seguimiento, 12 pacientes fallecieron y 7 fueron trasplantados. De los pacientes que permanecían en hemodiálisis tras un año de seguimiento, se excluyó a uno por desarrollar demencia. Todos aceptaron su participación voluntaria en el estudio.

Diseño del estudio y mediciones. El trabajo de campo fue realizado en el primer trimestre de 2004. Las entrevistas y la realización de cuestionarios se hicieron durante la hemodiálisis. Se recogieron datos clínicos, demográficos, uso de medicación, talla y peso. El estado de salud fue evaluado mediante la versión española del cuestionario SF-36 que contiene 36 items, que al cuantificarlos quedan agrupados en 8 dominios de salud. Cada dominio es puntuado en una escala de 0 a 100, de forma que una puntuación mayor indica un mejor estado de salud y viceversa ${ }^{3}$. Para determinar el grado de co-morbilidad de los pacientes y su potencial influencia sobre la calidad de vida de los mismos, se utilizó el Índice de Charlson ${ }^{4}$. El estado de ansiedad y/o depresión se evaluó mediante el cuestionario Hospital Anxiety-Depression ${ }^{5}$. Se trata de un cuestionario auto-administrado con 8 items referidos a la subescala depresión y otros 8 items referidos a la subescala ansiedad. Cada item adopta un valor de 0 a 3 en una escala tipo Likert de forma que la mínima y máxima puntuación para cada subescala es de 0 y 24 respectivamente. Un valor inferior a 7 es normal; los valores entre 8 y 10 son dudosos y los valores de 11 o superiores indican la presencia de ansiedad o depresión.

Análisis estadístico. Se comparó las diferencias entre los componentes de la CV, el test de ansiedad/depresión y el valor de la comorbilidad, mediante el análisis de t de una vía. Se utilizó un análisis de correlación simple de Pearson para conocer la relación entre el nivel de CV y variables independientes, conocidas previamente de nuestro estudio anterior, como el grado de comorbilidad y el estado depresivo. Se utilizó un análisis de regresión múltiple con los diferentes componentes del SF-36 como variables dependientes. En el modelo predictor se introdujeron los datos demográficos, el índice de comorbilidad y el valor del cuestionario de ansiedad-depresión independientemente de su peso individual. La incorporación de las variables se realizó paso a paso según su peso específico en la regresión lineal simple.

\section{RESULTADOS}

Características de los pacientes. La serie incluye 52 pacientes, 30 hombres y 22 mujeres. En la tabla 1 aparecen las principales características demográficas y clínicas de los pacientes vivos y del grupo de fallecidos a lo largo del año de seguimiento. La población viva sigue siendo ampliamente representativa, incluye un amplio rango de edad ( 26 a 78 años) y un tiempo en tratamiento en hemodiálisis variable ( 2 a 25 años). El estado nutricional fue disperso, incluyendo pacientes con franca delgadez (índice de masa corporal inferior a 20 $\mathrm{kg} / \mathrm{m}^{2}$ ) y clara obesidad (índice de masa corporal superior a 28 $\mathrm{kg} / \mathrm{m}^{2}$ ). No existieron diferencias entre hombres y mujeres respecto a la edad, tiempo en diálisis, estado nutricional o índice de comorbilidad. Como cabía esperar, el grupo de fallecidos era mas añoso respecto al grupo superviviente (70 \pm 43 versus $57 \pm 17$ años respectivamente, $\mathrm{p}<0.01)$ y con mas procesos de comorbilidad asociados $(4.8 \pm 2.4$ vs $3.5 \pm 2.4$, $\mathrm{p}<0.05)$.

Calidad de vida. El valor medio y el rango de cada escala de calidad de vida un año antes de la evaluación actual aparecen en la tabla 1 en función de su estado vital un año después. Puede observarse como no existían diferencias a ningún nivel de los componentes de la CV entre vivos y muertos. Tampoco existían diferencias entre el nivel de ansiedad y el de depresión. Al realizar el análisis de los diferentes componentes 
de la CV un año después del primer estudio, se pudo observar como la distribución de las puntuaciones para las escalas como limitación del rol físico, limitación del rol emocional y función social, no fueron gausianas; este comportamiento ya se identificó en el estudio inicial y entonces se explicó las razones. En esta ocasión tampoco existió diferencia para ninguna de las escalas de calidad de vida entre hombres y mujeres. En la figura 1 vienen expresadas los valores de las distintas escalas de calidad de vida en 2003 y 2004, en forma de medias y desviación típica. Las variaciones anuales de los distintos componentes de calidad de vida que evalúa el SF-36, han sido muy amplias y solo el componente de función física (FF) se ha deteriorado significativamente $(p<0.05)$. Incluso en este componente como puede apreciarse en la figura, existen variaciones individuales muy amplias, de forma que existieron pacientes con un deterioro espectacular que cayó a nivel de " 0 ", junto a otros pacientes que mostraron modificaciones a mejor o peor CV. En el análisis de regresión lineal simple la edad determinó de forma desfavorable la calidad de vida sólo en su componente de funcionalidad física $(r=0.41, p<0.01)$. Ningún otro parámetro estudiado se relacionó con el deterioro de los otros componentes del SF-36. Cuando analizamos la influencia de la comorbilidad, se encontró que solo el dominio de "funcionalidad física" mostró una relación negativa significativa con el nivel de depresión $(p<0.05)$ pero no estadísticamente significativa con el aumento de enfermedades concomitantes en pacientes con hemodiálisis (figura 2).

\begin{tabular}{|l|c|c|}
\hline Variable & Fallecidos $(\mathbf{n}=\mathbf{1 2})$ & Vivos $(\mathbf{n}=\mathbf{5 2})$ \\
\hline Edad, años & $70 \pm 43$ & $57 \pm 17^{* * *}$ \\
\hline IMC, Kg/m2 & $24.1 \pm 11$. & $23.8 \pm 5.9$ \\
\hline Sexo, H/M & $8 / 4$ & $30 / 22$ \\
\hline Tiempo en diálisis, años & $4.2 \pm 2.4$ & $3.9 \pm 1.1$ \\
\hline Índice de comorbilidad & $4.8 \pm 4.9$ & $3.5 \pm 2.4^{*}$ \\
\hline Funcionalidad física & $42.9 \pm 26.9$ & $49.9 \pm 31.3$ \\
\hline Limitación rol físico & $50.1 \pm 62.2$ & $52.9 \pm 42.5$ \\
\hline Dolor & $62.7 \pm 41.1$ & $62.2 \pm 29.1$ \\
\hline Funcionalidad social & $72.9 \pm 62.2$ & $69.7 \pm 33.2$ \\
\hline Estado mental & $59.1 \pm 48.1$ & $70.2 \pm 22.1$ \\
\hline Limitación rol emocional & $64.1 \pm 62.2$ & $79.1 \pm 39$ \\
\hline Vitalidad & $45.8 \pm 58.2$ & $52.3 \pm 25.6$ \\
\hline Percepción salud general & $39.2 \pm 23.3$ & $39.3 \pm 18.6$ \\
\hline Ansiedad & $6.75 \pm 7.8$ & $6.23 \pm 4.45$ \\
\hline Depresión & $7.6 \pm 4.9$ & $5.9 \pm 4.8$ \\
\hline & & \\
\hline ** $p<0.01 ;{ }^{*} p<0.05$ & & \\
\hline
\end{tabular}

TABLA 1. Características de los pacientes en hemodiálisis, fallecidos y vivos tras 1 año de seguimiento.
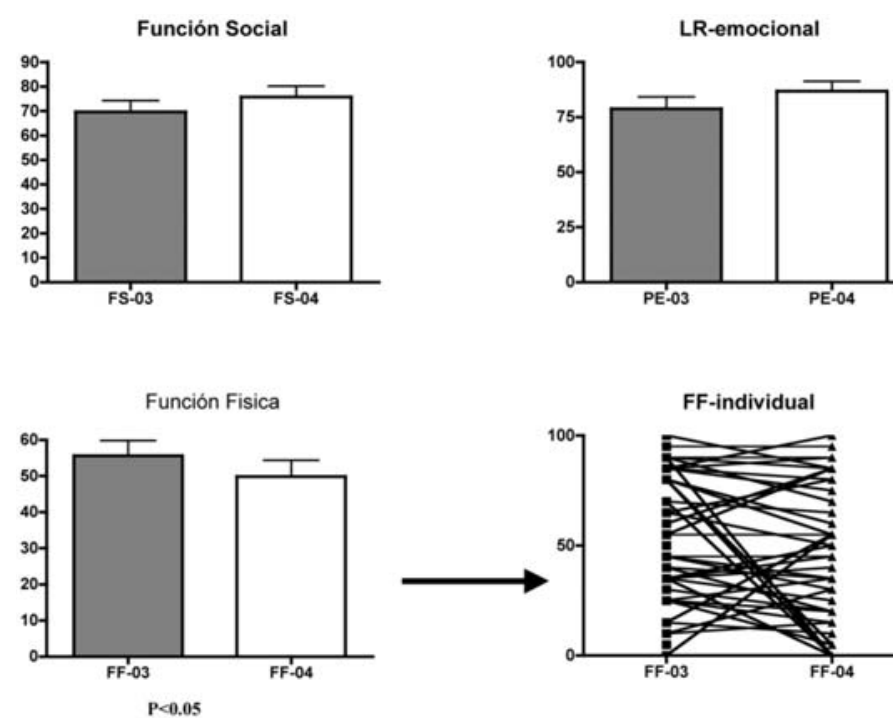

Figura 1. Cambios en la Calidad de Vida.

A
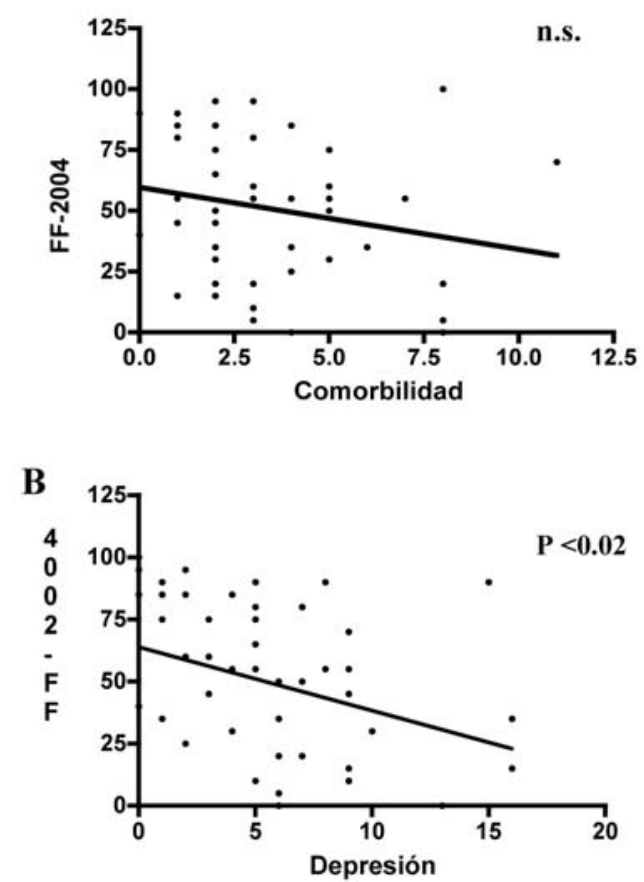

Figura 2. Relación de funcionalidad física con la comorbilidad y el nivel de depresión.

Comorbilidad y ansiedad/depresión. La comorbilidad al año de iniciado el estudio, aumentó de forma variable para la mayoría de pacientes aunque, como ya se ha indicado, el valor de la comorbilidad no determinó de forma significativa la $\mathrm{CV}$. Los valores del cuestionario de ansiedad-depresión indicaron la presencia de depresión (valor de la subescala $>11$ ) en un $7.7 \%$ de pacientes y de ansiedad (valor de la subescala $>$ 11) en un $7.7 \%$. Cuando se compara el estado psicológico en un año de diferencia, puede observarse en la figura 3 como nuevamente las variaciones son amplias. Para el conjunto del grupo, el estado de ansiedad se redujo de 6.75 a 6.23 de va- 
lores medios y el estado depresivo se redujo de 7.6 a 5.9 de valores medios; ambos cambios no son estadísticamente significativos. En el análisis de regresión múltiple la calidad de vida a nivel del dominio FF indicó, nuevamente un año después, que 3 variables introducidas paso a paso justifican esta vez un $67 \%$ de la varianza del valor de FF: edad, comorbilidad y grado de depresión.
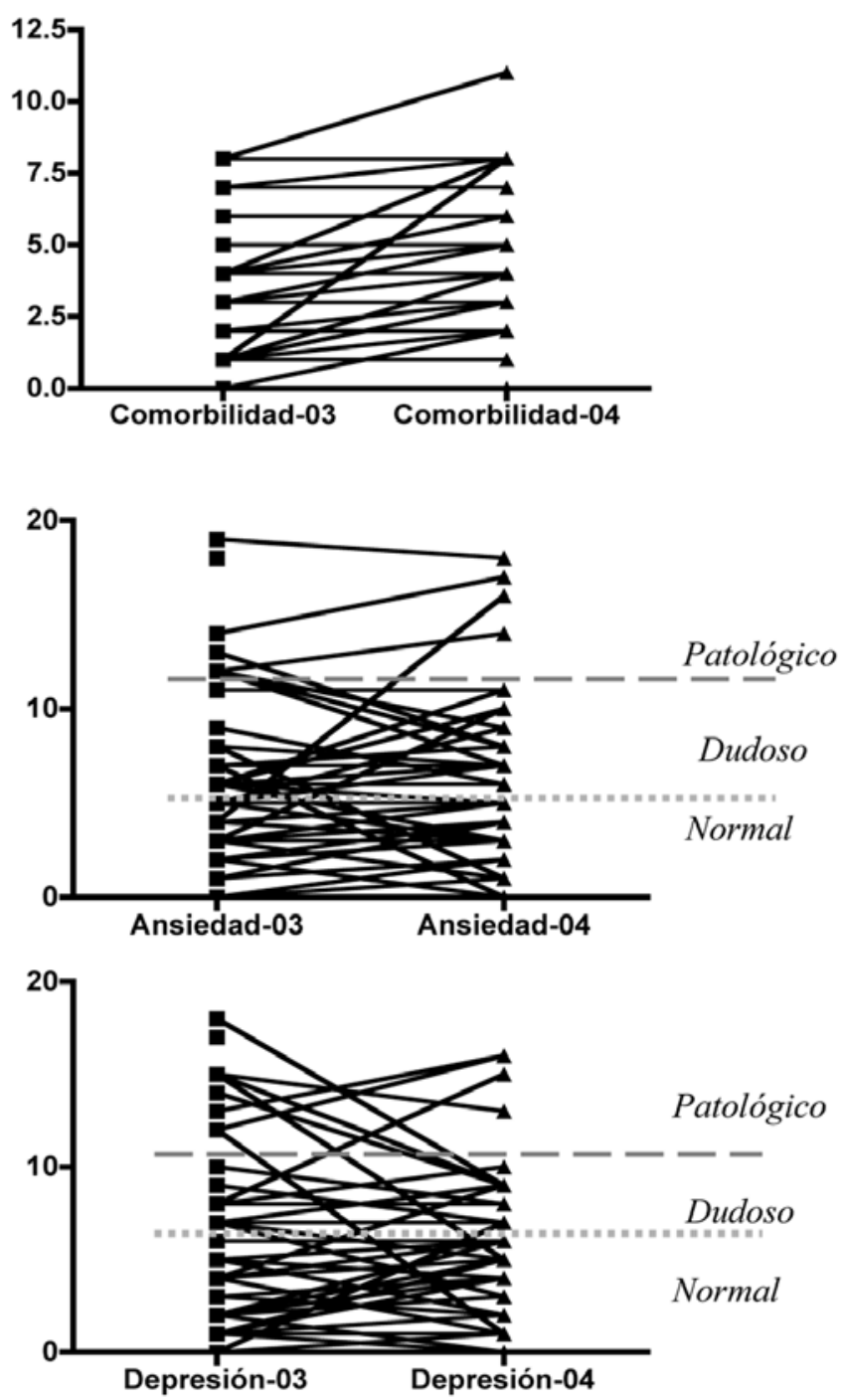

Figura 3. Cambios individuales en la comorbilidad y en el test de ansiedaddepresión.

\section{DISCUSIÓN}

Tras un año de seguimiento, los pacientes que permanecen en hemodiálisis muestran cambios muy sustanciales interindividuales e intraindividuales en la calidad de vida. Sólo el componente de función física se deteriora para el conjunto del grupo. La edad de los sujetos determina el componente de funcionalidad física y no es de extrañar, en este sentido, que este mismo dominio de la calidad de vida que explora la capacidad física de los sujetos, también se relacione de forma negativa con el índice de comorbilidad, ya que parece lógico esperar que con la edad aparezcan enfermedades clínicas asociadas tales como enfermedades vasculares y neoplásicas. Sigue siendo evidente la influencia del estado psicológico del paciente en la $\mathrm{CV}$, especialmente en su componente funcional. En este estudio se demuestra como el estado depresivo cambia en el tiempo, pero mantiene su influencia en la calidad de vida de los pacientes en hemodiálisis, sugiriendo en realidad, que tal vez el tratamiento en sí (las sesiones de hemodiálisis) tenga un peso menor que el propio estado depresivo del sujeto, para justificar el deterioro de la $\mathrm{CV}$ en pacientes en hemodiálisis respecto a la población general.

\section{BIBLIOGRAFÍA}

1. Oto Royo A, Muñoz Sancho R, Barrio Alonso R, Izuel Pérez $\mathrm{M}^{\mathrm{a}} \mathrm{L}$, Matud Aznar T. Calidad de vida en pacientes en pacientes en hemodiálisis: influencia del estado de ansiedad-depresión y de otros factores de comorbilidad. XXVIII Congreso Nacional de la SEDEN. Palma de Mallorca 2003.

2. Velasco Ballestero S, Álvarez Pérez R, San Juan Miguelsanz M. Sintomatología, trastornos emocionales y calidad de vida relacionada con la salud en pacientes en programa de hemodiálisis periódica. XXV Congreso Nacional de la SEDEN. Oviedo 2000.

3. Ayuso-Mateos JL, Lasa L, Vázquez-Barquero JL, Oviedo A, Diez-Maneique JF. Measuring health status in psychiatric community surveys: internal and external validity of the Spanish versión of the SF-36. Acta Psychiatr Scand 1999; 99:26.

4. Clarlson M, Szatrowski T, Peterson J, Gold J. Validation of a combinated index. J Clin Epidemiol 1994; 47:1245-1251.

5. Zigmond AS, Snaith RP. The Hospital Anxiety and Depression Scale. Acta Psychiatric Scand 1983; 67:361-370. 